\title{
Hippo promotes microRNA processing
}

MicroRNAs (miRNAs) are globally downregulated in human tumours, possibly as a result of reduced miRNA processing, and they might have a causative role in tumorigenesis, but the mechanisms controlling this are unknown. As both miRNA processing and the Hippo tumoursuppressor pathway are sensitive to cell density and contact inhibition, Fernando Camargo, Richard Gregory and colleagues investigated whether these two pathways are connected.

The processing of miRNAs

it challenges

our views

on the mechanisms

by which

Hippo

signalling

suppresses

tumours requires cleavage of primary miRNAs (pri-miRNAs) by the Microprocessor complex to form pre-miRNAs, which are then cleaved by Dicer complexes to form mature miRNAs. In the HaCaT keratinocyte cell line, miRNA levels were increased at high cell density, and the corresponding pri-miRNAs were decreased, whereas the converse was observed at low cell density, suggesting a densitydependent difference in miRNA

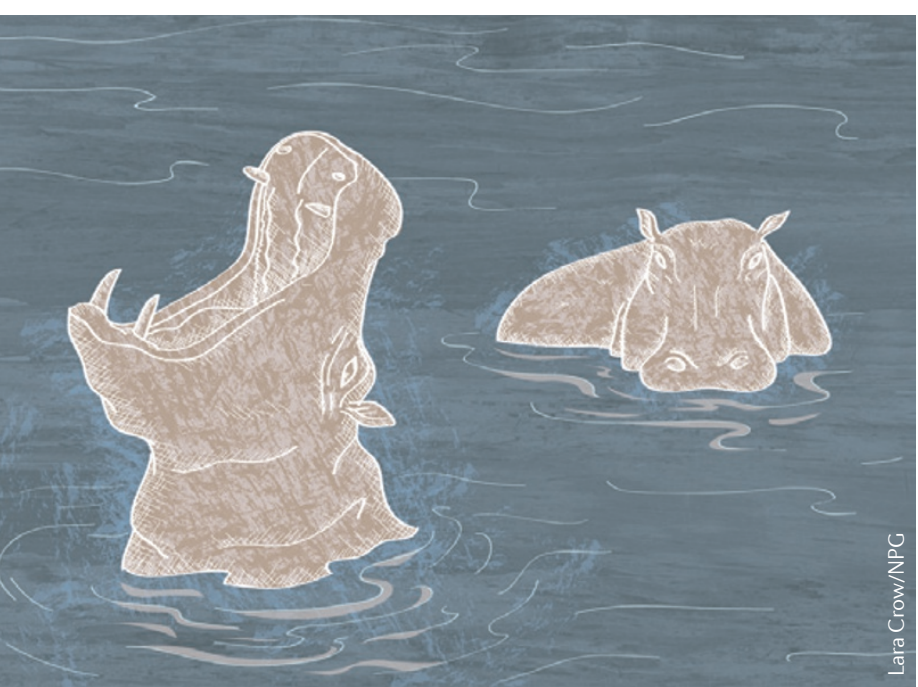

processing. A pri-miRNA luciferase reporter construct that is sensitive to Microprocessor activity was used to show that Microprocessor activity (but not Dicer activity) was activated at high cell density.

As cell-cell contacts form, Hippo signalling promotes translocation of the transcriptional co-activator Yesassociated protein (YAP) from the nucleus to the cytoplasm to repress proliferation, and various lines of evidence revealed that nuclear YAP can repress Microprocessor activity. The authors found that YAP can physically interact with p72, a Microprocessor component and RNA helicase, that p72 and YAP colocalize in the nucleus at low cell density and that suppression of Microprocessor activity requires YAP-p72 interaction. Translocation of YAP to the cytoplasm in response to Hippo signalling requires upstream activation of large tumour suppressor 2 (LATS2) and neurofibromin 2 (NF2; also known as Merlin), and knockdown of either of these proteins also impaired Microprocessor activity, which suggests that loss of Hippo signalling in cells at high density might globally repress miRNA levels. Profiling of more than 600 miRNAs revealed that approximately $60 \%$ of all expressed miRNAs were suppressed by NF2, LATS2 or p72 knockdown in highdensity HaCaT cells, and there was substantial overlap among those suppressed by $\mathrm{p} 72$ knockdown, by NF2 or LATS2 knockdown and by low cell density.

What is the potential relevance of this pathway in cancer? The authors analysed two mouse models in which tumours are driven by YAP. In the first model, a constitutively nuclear YAP mutant was expressed in the epidermis, leading to squamous cell carcinoma-like tumours. In the second model, NF2 was deleted in hepatocytes, leading to the formation of liver tumours. Tumour cells from both models showed an increase in pri-miRNAs (with a corresponding increase in YAP and p72 interaction) and a decrease in mature miRNAs compared with normal tissue. Using two different cell lines, the authors also showed that YAP binding to p72 promoted cell proliferation. YAP is primarily known as a regulator of transcription, but increased proliferation was independent of this function. MYC was one of the proteins induced post-transcriptionally through increased nuclear YAP and miRNA suppression, and this suggests that MYC might be responsible for some of the observed tumorigenic functions. Although MYC itself can repress miRNAs, it does so through preventing their transcription, so the accumulation of pri-miRNAs observed in this study provides an argument against this explanation.

Control of miRNA processing by the Hippo pathway might explain how this pathway suppresses proliferation in response to contact inhibition, and it challenges our views on the mechanisms by which Hippo signalling suppresses tumours.

Sarah Seton-Rogers

ORIGINAL RESEARCH PAPER Mori, M. et al. Hippo signaling regulates Microprocessor and links cell-density-dependent miRNA biogenesis to cancer. Cell 156, 893-906 (2014) 